PROCEEDINGS OF THE

AMERICAN MATHEMATICAL SOCIETY

Volume 128, Number 11, Pages 3205-3210

S 0002-9939(00)05508-8

Article electronically published on May 11, 2000

\title{
A PROPERTY OF SUBGROUPS OF INFINITE INDEX IN A FREE GROUP
}

\author{
G. N. ARZHANTSEVA
}

(Communicated by Ronald M. Solomon)

\begin{abstract}
We prove that if $H$ is a finitely generated subgroup of infinite index in a free group $F_{m}$, then, in a certain statistical meaning, the normal subgroup generated by "randomly" chosen elements $r_{1}, \ldots, r_{n}$ of $F_{m}$ has trivial intersection with $H$.
\end{abstract}

\section{INTRODUCTION}

Let $F_{m}$ be a free group with free generators $x_{1}, \ldots, x_{m}$ and $H$ a finitely generated subgroup of $F_{m}$. It is known 3] that if $H$ contains a non-trivial normal subgroup of $F_{m}$, then $H$ has finite index in $F_{m}$. Karrass and Solitar proved in 4 that if $H$ has non-trivial intersection with every non-trivial normal subgroup of $F_{m}$, then $H$ has finite index in $F_{m}$. This is a stronger result, since any two non-trivial normal subgroups of a free group have non-trivial intersection. On the other hand, it is obvious that if $H$ has finite index in $F_{m}$, then $H$ has non-trivial intersection with each of the non-trivial subgroups of $F_{m}$. Thus if $H$ is a finitely generated subgroup of $F_{m}$, then $H$ is of infinite index if and only if there is a normal subgroup $K$ of $F_{m}$ such that $K \cap H=\{1\}$. In the present paper, we study this property of subgroups of free groups from a statistical point of view. We prove that if $H$ is a finitely generated subgroup of $F_{m}$ of infinite index, then a randomly chosen normal subgroup $K$ of $F_{m}$ has trivial intersection with $H$ with the probability tending to 1 as the lenghts of the elements whose normal closure is $K$ tend to infinity. In other words, this gives a "generic property" of normal subgroups of a free group: for a fixed $H$, a generic normal subgroup of $F_{m}$ trivially intersects with $H$.

For a precise formulation, let $N=N(m, n, t)$ denote the number of all $n$-tuples $\left\{r_{1}, \ldots, r_{n}\right\}$ of cyclically reduced words in the alphabet $X_{m}=\left\{x_{1}^{ \pm 1}, \ldots, x_{m}^{ \pm 1}\right\}$ of length $\left|r_{i}\right| \leq t$ and let $N_{H}=N_{H}(m, n, t)$ denote the number of all such $n$-tuples $\left\{r_{1}, \ldots, r_{n}\right\}$ whose normal closure in $F_{m}$ has trivial intersection with $H$. Our main result is the following theorem.

Received by the editors January 15, 1999.

2000 Mathematics Subject Classification. Primary 20E07, 20F06, 20 P05.

Key words and phrases. Free groups, generic properties of groups, labelled graphs.

The work is supported by the Russian Foundation for Fundamental Research grant 96-01-0420 and by ISSEP grant a98-2146. 
Theorem 1. For any fixed $n$ and $m$, if $H$ is a finitely generated subgroup of infinite index in $F_{m}$, then

$$
\lim _{t \rightarrow \infty} N_{H} / N=1 .
$$

Moreover, there is a real number $c>0$ depending on $m, n$ and $H$ such that $1-N_{H} / N<\exp (-c t)$ for all $t>0$.

Note that the normal closure of the elements $r_{1}, \ldots, r_{n}$ depends only on their conjugacy classes. So the requirement for $r_{i}$ to be cyclically reduced is not restrictive in this sense.

In the proof of Theorem 1, we will use presentations of finitely generated subgroups of $F_{m}$ by finite connected labelled graphs. Such presentations have been introduced by J. Stallings and have been used to study subgroups of free groups, free inverse monoids, and "statistical" properties of finitely presented groups (for details see [7, [6] and [1]).

Using Theorem 1 we get a generic property of finitely presented groups. Recall the definition of a generic property. For any $m \geq 2$ and $n \geq 1$ we consider all group presentations

$$
G=\left\langle x_{1}, \ldots, x_{m} \mid r_{1}=1, \ldots, r_{n}=1\right\rangle,
$$

where $r_{1}, \ldots, r_{n}$ are cyclically reduced words in the alphabet $X_{m}$ of length $\left|r_{i}\right| \leq t$. Note that the number of all such presentations (1I) is exactly $N=N(m, n, t)$. Let $N_{\mathcal{P}}$ be the number of presentations (11) with the group $G$ having a property $\mathcal{P}$. A property $\mathcal{P}$ of $m$-generated groups is said to be generic if $\lim _{t \rightarrow \infty} N_{\mathcal{P}} / N=1$ for any $n$. We say that the genericity is exponential if this ratio tends to 1 faster than some function $1-\exp (-c t)$ with $c>0$. In this paper, we will consider exponential genericity only. For more information and examples of generic properties see [1], [2].

Theorem 2. Let $h_{1}, h_{2}, \ldots, h_{k}$ be elements of the free group $F_{m}$ and $r \geq 1$. Then the following conditions are equivalent:

(i) the elements $h_{1}, h_{2}, \ldots, h_{k}$ generate a free subgroup of rank $r$ of infinite index in $F_{m}$;

(ii) for a generic group $G$, the images of the elements $h_{i}$ under the natural epimorphism $F_{m} \rightarrow G$ generate a free subgroup of rank $r$ in $G$;

(iii) for a generic group $G$, the images of the elements $h_{i}$ under the natural epimorphism $F_{m} \rightarrow G$ generate a free subgroup of rank $r$ of infinite index in $G$.

\section{GraphS AND SUbGroups}

Let $\Gamma$ be a graph. By an edge of $\Gamma$ we mean here a directed edge, i.e., an edge of $\Gamma$ in the usual sense with any of its two possible directions. If $e$ is an edge of $\Gamma$, then $e^{-1}$ denotes the edge with the opposite direction. A map $\varphi$ from the edges of $\Gamma$ to $X_{m}$ is called a labelling function on $\Gamma$ if it satisfies $\varphi\left(e^{-1}\right)=(\varphi(e))^{-1}$ for any edge $e$. By the label $\varphi(p)$ of a path $p=e_{1} e_{2} \ldots e_{k}$ of length $k$ in $\Gamma$ we mean the word $\varphi\left(e_{1}\right) \varphi\left(e_{2}\right) \ldots \varphi\left(e_{k}\right)$. The label of a path of length 0 (which by definition is identified with a vertex of $\Gamma$ ) is the empty word.

A labelled graph is a finite connected graph $\Gamma$ with a labelling function $\varphi$ and a distinguished vertex $O$. Any labelled graph $\Gamma$ represents a subgroup $H(\Gamma)$ of $F_{m}$, which is the image of the fundamental group $\pi_{1}(\Gamma, O)$ under the homomorphism 
induced by $\varphi$. In other words, $x \in H(\Gamma)$ if and only if $x$ may be represented by a word which can be read on a circuit at $O$.

It is easy to see that any finitely generated subgroup $H \subset F_{m}$ may be represented by a labelled graph. To do this, we first take words $h_{1}, h_{2}, \ldots, h_{k}$ in the alphabet $X_{m}$ that represent generators of $H$. Next we take a rose of $k$ circles attached to a point $O$ and make each of the circles a circuit labelled $h_{i}, 1 \leq i \leq k$. For the resulting labelled graph $\Gamma$, we obviously have $H(\Gamma)=H$.

We define two types of transformations of a labelled graph $\Gamma$, which preserve the subgroup $H(\Gamma)$ and which we call reductions. A transformation of the first type is identification of two edges with the same label and the same initial vertex. A transformation of the second type is removal of a vertex of degree 1 other than $O$, together with the incoming edge.

A labelled graph $\Gamma$ is said to be reduced if it admits no reductions; that is, it has no pair of edges with the same label and initial vertex and no vertices of degree 1 with the possible exception of the distinguished vertex $O$.

Starting from a labelled graph $\Gamma$ with $H(\Gamma)=H$ and performing all possible reductions, we reach a reduced labelled graph which represents the subgroup $H$. It is known [7, [6] that a reduced labelled graph representing a subgroup $H$ is unique up to graph isomorphism (that is, it does not depend on the order of reductions, the choice of the initial graph $\Gamma$, and the choice of generators for $H$ ).

If $\Gamma$ is a reduced labelled graph, then it is easy to see that a reduced word $w$ represents an element of $H(\Gamma)$ if and only if $w$ is the label of a reduced circuit at $O$ in $\Gamma$. It follows in particular that the label of a path $p$ in $\Gamma$ starting at $O$ represents an element of $H(\Gamma)$ only if $O$ is also the terminal vertex of $p$.

Lemma. Let $F_{m}$ be a free group with $m$ generators, $H$ a finitely generated subgroup of $F_{m}$, and $\Gamma$ a reduced labelled graph representing the subgroup $H$.

Then the degree of any vertex of $\Gamma$ is maximal (i.e., equals $2 m$ ) if and only if $H$ has finite index in $F_{m}$.

In addition, if $H$ has finite index, the number of vertices of $\Gamma$ is equal to the index $\left|F_{m}: H\right|$.

Since the graph $\Gamma$ is reduced, the maximal degree of any vertex of $\Gamma$ is $2 m$.

Suppose each vertex of $\Gamma$ has degree $2 m$. This means that, for any vertex $v$ of $\Gamma$ and any letter $x \in X_{m}$, there is exactly one edge starting at $v$ and labelled $x$. Then it is easy to see that $F_{m}$ acts transitively on the vertices of $\Gamma$ in the following way. An element $w \in F_{m}$ sends a vertex $v$ of $\Gamma$ to the terminal vertex of the path starting at $v$ and labelled $w$. The stabilizer of the distinguished vertex $O$ is exactly the subgroup $H$. Hence the index of $H$ is the number of vertices of $\Gamma$ and so it is finite.

Now suppose that a vertex $v$ of $\Gamma$ has non-maximal degree. This means that for some letter $x \in X_{m}$, there is no edge starting at $v$ labelled $x$. Let $w$ be the label of any path joining $O$ with $v$. By the assumption, there is no reduced path in $\Gamma$ starting at $O$ with label $w x^{i} w^{-1}$ for $i>0$. Hence $w x^{i} w^{-1} \notin H$ which easily implies that $H$ has infinite index in $F_{m}$.

\section{Proof of Theorem 1}

Let $\left\{r_{1}, \ldots, r_{n}\right\}$ be an $n$-tuple of cyclically reduced words in the alphabet $X_{m}$ of length $\left|r_{i}\right| \leq t$ and let $\Gamma$ be a reduced labelled graph representing $H$. It is easy to see 
that the number of cyclically reduced words $r$ in $X_{m}$ of length $|r| \leq l$ is greater than $(2 m-1)^{l-1}$ but less than $1+2 m+2 m(2 m+1)+\cdots+2 m(2 m-1)^{l-1}<2(2 m-1)^{l}$.

Let us make a few observations.

I. Let $R_{*}\left(r_{1}, \ldots, r_{n}\right)$ denote the set containing all cyclic shifts of words $r_{i}$ and their inverses. Note that the share of all $n$-tuples $\left\{r_{1}, \ldots, r_{n}\right\}$ so that $R_{*}\left(r_{1}, \ldots, r_{n}\right)$ does not satisfy the small cancellation condition $C^{\prime}\left(\frac{1}{6}\right)$ decreases exponentially as $t \rightarrow \infty$ (see [1]). Hence to prove the theorem we may suppose that $R_{*}\left(r_{1}, \ldots, r_{n}\right)$ satisfies $C^{\prime}\left(\frac{1}{6}\right)$.

II. We may assume that $\left|r_{i}\right|>\frac{t}{2}$ for all $i$.

Indeed, the number $N(m, n, t)$ of all $n$-tuples of cyclically reduced words in the alphabet $X_{m}$ of length $\leq t$ is greater than $(2 m-1)^{n(t-1)}$. On the other hand, the number of $n$-tuples $\left\{r_{1}, \ldots, r_{n}\right\}$ such that $\left|r_{i}\right| \leq t$ for all $i$ and $\left|r_{i}\right| \leq t / 2$ for at least one $i$ does not exceed $n 2^{n}(2 m-1)^{(n-1) t+t / 2}$. Clearly, the last value is exponentially smaller than $N(m, n, t)$ as $t$ tends to infinity.

III. We may assume that any cyclic shift of any $r_{i}$ contains no subword of length $\geq \frac{\left|r_{i}\right|}{2}$ which is the label of a path of $\Gamma$.

By II, we may assume that $\left|r_{i}\right| \geq t / 2$ for all $i$. We estimate the number of words of length $\left[\frac{t}{4}\right]$ which are labels of reduced paths in $\Gamma$ starting at an arbitrary fixed vertex. By the lemma, $\Gamma$ has a vertex of non-maximal degree. Let $d$ be greater than the diameter of $\Gamma$, that is, any two vertices of $\Gamma$ are joined with a path of length $<d$. When counting the number of reduced paths of length $d$, at least one way for extension of a path does not exist at the vertex of non-maximal degree. Then there are at most $2 m(2 m-1)^{d-1}-1$ reduced paths of length $d$ in $\Gamma$ starting at any fixed vertex of $\Gamma$. Representing $\left[\frac{t}{4}\right]$ as $d k+s$ with $0 \leq s<d$, we see that there are at most

$$
\left(2 m(2 m-1)^{d-1}-1\right)\left((2 m-1)^{d}-1\right)^{k-1}(2 m-1)^{s}
$$

reduced words of length $\left[\frac{t}{4}\right]$ which can be read on paths in $\Gamma$ with a given initial vertex. From this it easily follows that the number of words $r_{i}$ of length $\left|r_{i}\right| \leq t$ such that a cyclic shift of $r_{i}$ has a subword of length $\left[\frac{t}{4}\right]$ which can be read on $\Gamma$ is at most

$$
V(H) t\left(2 m(2 m-1)^{d-1}-1\right)\left((2 m-1)^{d}-1\right)^{k-1}(2 m-1)^{s} 2(2 m-1)^{\frac{3 t}{4}+1},
$$

where $V(H)$ is the number of vertices of $\Gamma$. Therefore the number of $n$-tuples containing at least one excluded word $r_{i}$ (that is, a label of a path of length $\geq\left[\frac{\left|r_{i}\right|}{2}\right]$ in $\Gamma$ is a subword of a cyclic shift of $r_{i}$ ) is at most

$$
\begin{gathered}
V(H) \operatorname{tn}\left(2 m(2 m-1)^{d-1}-1\right)\left((2 m-1)^{d}-1\right)^{k-1}(2 m-1)^{s}(2 m-1)^{\frac{3 t}{4}+1} \\
\times\left(2(2 m-1)^{t}\right)^{n-1} .
\end{gathered}
$$

Then the share of such $n$-tuples among all $n$-tuples of cyclically reduced words is at most

$$
\frac{\operatorname{Btn}\left((2 m-1)^{d}-1\right)^{k}(2 m-1)^{\frac{3 t}{4}+1}}{(2 m-1)^{t}}
$$


where $B=B(m, n, H)$ is a constant. Since $t \geq\left[\frac{t}{4}\right]+\frac{3 t}{4}$, we obtain that this ratio is at most

$$
C t\left(1-\frac{1}{(2 m-1)^{d}}\right)^{\left[\frac{t}{4}\right] / d},
$$

where a constant $C=C(m, n, H)$ is independent of $t$. This value decreases exponentially as $t \rightarrow \infty$.

Thus for the proof of the theorem we may assume that the $n$-tuple $\left\{r_{1}, \ldots, r_{n}\right\}$ satisfies conditions I-III. Assume that $H \cap \mathcal{N}\left(r_{1}, \ldots, r_{n}\right) \neq 1$, where $\mathcal{N}\left(r_{1}, \ldots, r_{n}\right)$ denotes the normal closure of the elements $r_{i}$ in $F_{m}$. Then there exists a non-empty reduced word $w$ which can be read on some circuit of $\Gamma$ starting at the distinguished vertex and equal to 1 in the group $F_{m} / \mathcal{N}\left(r_{1}, \ldots, r_{n}\right)$. By Condition I and the Greendlinger lemma for $C^{\prime}\left(\frac{1}{6}\right)$-groups (see [5]), the word $w$ contains a subword $v$ that is also a subword of a cyclic shift of some $r_{i}^{ \pm 1}$ and satisfies $|v|>(1-3 \lambda)\left|r_{i}\right|$. Hence we obtain $|v|>\frac{\left|r_{i}\right|}{2}$ and $v$ can be read on a path of $\Gamma$. But this contradicts to Condition III.

\section{A GENERIC PROPERTY OF GROUPS}

In the proof of Theorem 2 we will use

Theorem 3 ([2]). For any given integer $L \geq 1$ and $m \geq 2$, the class of m-generated groups all of whose subgroups of infinite index with at most $L$ generators are free but all subgroups of finite index are not free is generic.

Proof of Theorem 2. Obviously, we need to prove only that (i) implies (iii) and (ii) implies (i).

Suppose that $h_{1}, h_{2}, \ldots, h_{k}$ generate a free subgroup $H$ of rank $r$ of infinite index in $F_{m}$. By Theorem 10 the normal closure $\mathcal{N}\left(r_{1}, \ldots, r_{n}\right)$ of "randomly" chosen elements $r_{1}, \ldots, r_{n}$ of $F_{m}$ has trivial intersection with $H$. Hence the restriction on $H$ of the natural epimorphism $F_{m} \rightarrow G$ with $G=F_{m} / \mathcal{N}\left(r_{1}, \ldots, r_{n}\right)$ is an isomorphism. Thus the images of the elements $h_{i}$ under the epimorphism generate a free subgroup $\bar{H}$ of rank $r$ in a generic $m$-generated finitely presented group. Theorem 3 implies in particular that all subgroups of finite index in a generic finitely presented group are not free. Therefore the index of $\bar{H}$ cannot be finite.

Now suppose that the images of the elements $h_{i}$ under the epimorphism $F_{m} \rightarrow G$ generate a free subgroup of rank $r$ of a generic finitely presented group $G$. Using Theorem 3 again, we see that this subgroup has infinite index. Obviously, the elements $h_{1}, h_{2}, \ldots, h_{k}$ generate then a subgroup of infinite index in $F_{m}$. This subgroup is of rank $r$, since we have already proved that (i) implies (iii).

Let us call a finite set of words in the alphabet $X_{m}$ admissible if the corresponding elements of the free group $F_{m}$ generate a subgroup of infinite index in $F_{m}$.

Note that there is an effective procedure to check whether a given finite set of words is admissible or not. First, given words $h_{1}, h_{2}, \ldots, h_{k}$, we construct a labelled graph representing the subgroup $\left\langle h_{1}, h_{2}, \ldots, h_{k}\right\rangle$ taking for example a rose of $k$ circuits each labelled with the corresponding $h_{i}$. Then we obtain a reduced labelled graph using reductions. By the lemma, the set $\left\{h_{1}, h_{2}, \ldots, h_{k}\right\}$ is admissible if and only if the corresponding reduced labelled graph has a vertex of degree $<2 m$. 
The following sets of words are admissible:

1) $\left\{x_{1}^{2}, x_{2}, \ldots, x_{m}\right\}$;

2) any finite set of words representing elements of the commutator subgroup of $F_{m}$.

Obviously, the following sets of words are not admissible:

1) $\left\{x_{1}, x_{2}, \ldots, x_{m}\right\}$;

2) $\left\{x_{i} x_{j}, x_{i}^{2}, x_{i} x_{j}^{-1}, x_{i}^{-1} x_{j} \mid 1 \leq i, j \leq m\right\}$ (this set generates the subgroup of $F_{m}$ of words of even length).

Now the previous theorem implies

Corollary. Let $S$ be any admissible set of words in the alphabet $X_{m}$. Then for any $m \geq 2$ and $n \geq 1$ the class of those $m$-generated groups with $n$ defining relations in which $S$ generates a free subgroup is exponentially generic.

\section{REFERENCES}

[1] G. N. Arzhantseva and A. Yu. Ol'shanskii, Generality of the class of groups in which subgroups with a lesser number of generators are free, Mat. Zametki, 59(4) (1996), 489-496 (in Russian), English translation in Math. Notes, 59(4) (1996), 350-355. MR 98k:20040

[2] G. N. Arzhantseva, On the groups all of whose subgroups with fixed number of generators are free, Fundamental and Applied Mathematics, 3(3) (1997), 675-683 (in Russian).

[3] L. Greenberg, Discrete groups of motions, Canad. J. Math., 12 (1960), 414-425. MR 22:5932

[4] A. Karrass and D. Solitar, On finitely generated subgroups of a free group, Proc. Amer. Math. Soc., 22 (1969), 209-213. MR 39:6961

[5] R. Lyndon and P. Schupp, Combinatorial Group Theory, Springer-Verlag, 1977. MR 58:28182

[6] S. W. Margolis and J. C. Meakin, Free inverse monoids and graph immersions, Int. J. Algebra and Comput., 3(1) (1993), 79-99. MR 94c:20105

[7] J. Stallings, Topology of finite graphs, Inv.Math., 71 (1983), 552-565. MR 85m:05037a

Section de Mathématiques, Université de Genève, CP 240, 1211 Genève 24, SwitzerLAND

E-mail address: Goulnara.Arjantseva@math.unige.ch 